Neue Wiederbelebungsrichtlinien für stark unterkühlte Unfallopfer

\title{
Höhere Überlebenschancen
}

Bei Unfallopfern mit Herzstillstand gilt allgemein die Regel, dass die begonnenen Wiederbelebungsmaßnahmen nicht unterbrochen werden dürfen, bis der Patient wieder Lebenszeichen aufweist oder der Tod festgestellt wird. Eine neue Studie des Bozner Forschungszentrums EURAC und der Medizinischen Universität Innsbruck zeigt, dass im spezifischen Fall von stark unterkühlten Unfallopfern die Herzdruckmassage für den Transport im Gebirge immer wieder kurz unterbrochen werden kann, ohne das Überleben zu gefährden.

In ausgesetztem Gelände stehen Bergretter bei der Behandlung von Patienten im Herzkreislaufstillstand oft vor dem Dilemma, dass eine lückenlose Wiederbelebung während des Transportes in ein Krankenhaus nicht möglich ist. In den Alpen gab es in den vergangenen Jahren einige Fälle von stark unterkühlten Unfallopfern mit Herzstillstand, die trotz mehrmals unterbrochener Herzdruckmassage ohne bleibende Schäden überlebt haben. Rettungsdienste drängen seit Längerem auf eine Klärung des Sachverhaltes speziell für Unterkühlungsopfer im Herzstillstand, da die derzeitige Regelung ausnahmslos eine lückenlose Wiederbelebung vorschreibt.

In dieser Studie kommen die Forscher zu dem Ergebnis, dass bei der Wiederbelebung von stark unterkühlten Patienten (Körperkerntemperatur unter $28^{\circ} \mathrm{C}$ ) kurze Pausen bei der Herzdruckmassage zugunsten des Transports gemacht werden können, ohne dass das Überleben dadurch in Gefahr gebracht wird oder mehr Hirnschäden auftreten. Die konservierende Wirkung der Kälte führt dazu, dass der Körper bei tiefen Körpertemperaturen einen Herzstillstand wesentlich länger toleriert als bei normaler Körpertemperatur.

\section{Die Herz- und Gefäßchirurgen machen's vor}

Zustand ist eine kurzzeitige Unterbrechung des Kreislaufs möglich, um die Eingriffe am Herzen oder an den großen herznahen Gefäßen durchführen zu können, ohne bleibende Hirnschäden befürchten zu müssen. „Wir haben in unserer Studie zum einen umfassende Fallanalysen durchgeführt, zum anderen aber auch von der Herzchirurgie gelernt. Wir schlagen daher vor, dass Retter und Notärzte bei schwer unterkühlten Patienten die Herzdruckmassage zur Wiederbelebung für den Transport kurzzeitig unterbrechen", erklären Priv.-Doz. Dr. Peter Paal von der Medizinischen Universität Innsbruck und Priv.Doz. Dr. Hermann Brugger vom EURAC-Institut für Alpine Notfallmedizin. „In der Praxis heißt das: Wenn schwer unterkühlte Patienten mit Herzstillstand aus unwegsamem Gelände evakuiert werden müssen und eine kontinuierliche Wiederbelebung nicht möglich ist, kann man abwechselnd fünf Minuten reanimieren, fünf Minuten transportieren und dies solange im Wechsel, bis wieder eine kontinuierliche Herzdruckmassage möglich ist. Somit können Patienten aus entlegenen Gebieten unter Wiederbelebung zur Wiedererwärmung in ein Krankenhaus mit Herzlungenmaschine gebracht und müssen nicht aufgegeben werden", so die Forschergruppe.

Das Ergebnis der Studie schafft die Grundlage für einen Paradigmenwechsel innerhalb der bislang gültigen Rettungsrichtlinien. Im Laufe dieses Jahres sollen die Erkenntnisse in die Richtlinien der Internationalen Kommission für Al-

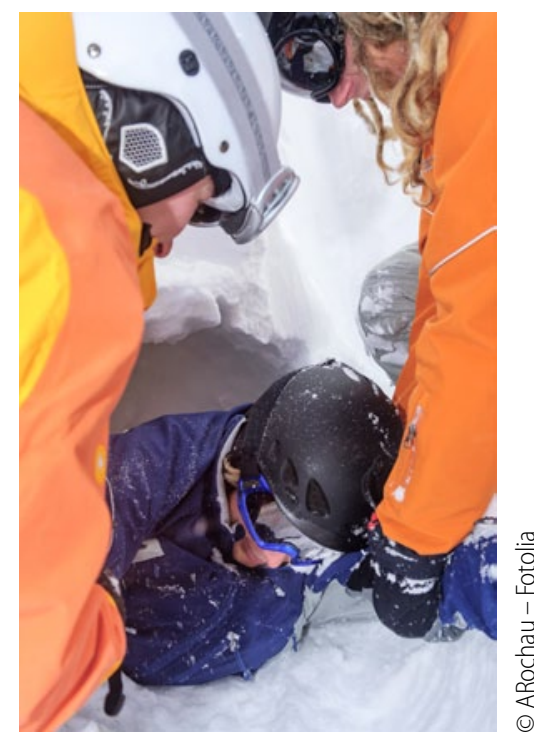

pine Notfallmedizin (ICAR Medcom) und des Europäischen Rates für Wiederbelebung (European Resuscitation Council, ERC) aufgenommen werden.

Literatur

1. Gordon L, Paal P, Ellerton JA, Brugger H, Peek GJ, Zafren K (2015) Delayed and intermittent CPR for severe accidental hypothermia. Resuscitation 90:46-49

Wien klin Mag 2015 · 18:182

DOI 10.1007/s00740-015-0073-2

Online publiziert: 1. Oktober 2015

C Springer-Verlag Wien 2015 